\title{
Genuine multipartite Einstein-Podolsky-Rosen steering
}

\author{
Q. Y. He $\mathrm{He}^{1,2}$ and M. D. Reid ${ }^{1, *}$ \\ ${ }^{1}$ Centre for Quantum Atom Optics, \\ Swinburne University of Technology, \\ Melbourne, 3122 Australia \\ ${ }^{2}$ State Key Laboratory of Mesoscopic Physics, \\ School of Physics, Peking University, Beijing 100871 China \\ *mdreid@swin.edu.au
}

\begin{abstract}
We develop the concept of genuine $N$-partite Einstein-Podolsky-Rosen (EPR) steering. This nonlocality is the natural multipartite extension of the original EPR paradox. Useful properties emerge that are not guaranteed for genuine multipartite entangled states. In particular, there is a close link with the task of one-sided device-independent quantum secret sharing. We derive inequalities to demonstrate multipartite EPR steering for Greenberger-Horne-Zeilinger (GHZ) and Gaussian continuous variable (CV) states in loophole-free scenarios.
\end{abstract}

Bell's seminal work showed that quantum mechanics is not equivalent to any local hidden variable theory (LHV) [1], but this work was a study of nonlocality between two particles only. Svetlichny asked whether quantum mechanics could exhibit a genuine three-body nonlocality [2], in which case the nonlocality cannot be simulated by any nonlocality that might exist between only two bodies. These ideas are crucial to understanding the full nature of the transition from the quantum to the classical regime [3 8, 10]. Seevinck, Collins and co-workers [5, 6] revealed that $N$-party Greenberger-Horne-Zeilinger (GHZ) states can exhibit genuine Bell nonlocality among $N$ sites, and experiments have reported violation of Svetlichny inequalities using GHZ states [9, 10]. The experimental violation however was limited to $N=3$, and to systems of only one qubit (photon) per site.

Our knowledge of multipartite entanglement on the other hand is much more established. Experimental signatures have been developed, for both continuous variable (CV) [11] and qubit systems [12 15]. There has been experimental evidence in both cases [14, 16, 17], with the generation of fourteen entangled qubits in iontraps [18] and recent reports of CV entanglement of up to eight light modes [19]. However, entanglement does not demonstrate nonlocality [20 22], and it is widely appreciated that the detection of Bell nonlocality is far more challenging [23]. Whether the observation of genuine $N$ partite Bell nonlocality is possible for systems of very high dimension or for $\mathrm{CV}$ measurements is not yet fully understood [3]. Despite this, there is an increasing awareness that nonlocality is not only fundamentally significant, but can be specifically required for certain quantum information tasks [13, 21, 22, 24 27].

In this Letter, we investigate an intermediate type of genuine $N$-body nonlocality. As it is potentially less susceptible to noise and decoherence than Bell nonlocality, it is therefore more accessible to experiment. We consider genuine multipartite forms of Einstein-Podolsky-Rosen (EPR) steering. Steering has only recently been identified as a distinct type of nonlocality [21, 22, 28], dif- ferent to both entanglement and Bell's nonlocality, and is realised in experiments that reveal an EPR paradox [29, 30]. Work by Wiseman and co-workers [21, 22] formalised Schrodinger's concept of "steering", that an observer can apparently instantaneously influence a distant system, by making local measurements. Multipartite EPR steering has been studied for qubits [31] and qudits [32]. However, this work did not examine genuine multipartite nonlocality, in which the nonlocality is necessarily shared among all observers.

We show that it is possible to obtain genuine multipartite EPR steering in very different sorts of systems to those so far predicted for multipartite Bell nonlocality. To date, EPR steering has been verified at very high detection efficiencies for CV Gaussian optical systems 30, 33, 34] and without detection loopholes for photons [35 37], but the focus has been on the bipartite case.

Here, we formalise the meaning of genuine multipartite EPR steering, and derive criteria to detect it. We show how to verify $N$-partite steering for GHZ states, both in discrete and CV Gaussian systems, giving efficiency bounds to do so conclusively. Our work therefore opens up possibilities to demonstrate an $N$-partite EPR nonlocality $(N>2)$ unambiguously for qubit sites, whether by using photons [10, 35, 37] or ions [16, 18], and to test the existence of the strongest form of nonlocality so far predicted to distribute over many sites with systems in the continuous (CV) limit. Further, we prove results for multipartite EPR steering, that are useful to multi-party quantum communication protocols [39 41], and are not guaranteed by multipartite entanglement.

Genuine $N$-partite nonlocality: We consider $N$ spatially separated systems at sites $j=1, \ldots, N$, and ask how to derive criteria for genuine $N$-party nonlocality, so that we can conclude nonlocality to be shared among all $N$ parties. The strongest form of nonlocality is Bell's nonlocality, in which all Local Hidden Variable (LHV) models are falsified [1]. Denoting the hidden variables that specify the predetermined nature of the system by the set $\{\lambda\}$, all LHV theories will imply the fully sepa- 
rable LHV model $\left\langle\prod_{j=1}^{N} X_{j}\right\rangle=\int_{\lambda} d \lambda P(\lambda) \prod_{j=1}^{N}\left\langle X_{j}\right\rangle_{\theta_{j}, \lambda}$. The $j-1$ factorisations in the integrand are justified based on the assumptions of locality and no-signalling between all sites $j$. Here $X_{j}$ are the possible results for a measurement $\hat{X}_{j}$ at site $j,\left\langle X_{j}\right\rangle_{\theta_{j}, \lambda}$ is the expected value of $X_{j}$ for a given set $\{\lambda\}$ where $\theta_{j}$ denotes the choice of measurement at site $j$, and $P(\lambda)$ is the hidden variable probability distribution function. Bell's nonlocality is demonstrated when the LHV model fails.

Genuine $N$-party Bell nonlocality can be tested using a method pioneered by Svetlichny [2, 5, 6]. We construct a hybrid local-nonlocal model in which Bell nonlocality exists, but only if shared among $N-1$ or fewer parties. Thus, the fully separable LHV model becomes only partially separable, with separability retained between any two groups $A$ and $B$ of $N-k$ and $k(k \leq N / 2)$ parties respectively. We label the possible ways of splitting the sites into two such groups by the index $s$. The Svetlichny model is

$$
\begin{aligned}
\left\langle\prod_{j=1}^{N} X_{j}\right\rangle= & \sum_{s} P_{s} \int_{\lambda} d \lambda P_{s}(\lambda) \\
& \left\langle\prod_{j \in A_{s}} X_{j}\right\rangle_{\left\{\theta_{j}\right\}, \lambda} \times\left\langle\prod_{j \in B_{s}} X_{j}\right\rangle_{\left\{\theta_{j}\right\}, \lambda}
\end{aligned}
$$

where $\sum_{s} P_{s}=1$. Failure of (1) implies genuine $N$ partite Bell nonlocality. Recently, Gallego et al [7] and Bancal et al [8] have revealed that the Svetlichny definition, which assumes fully "bi-local" (BL) correlations, is strictly stronger than necessary to confirm genuine $N$ partite nonlocality. Surprisingly, the bipartition $\left\{A_{s}, B_{s}\right\}$ can admit a Bell nonlocality, if all types of signalling between the parties of a group are allowed. By relaxing the assumptions of the model, to allow only "time-ordered bi-local correlations" (TOBL), a more sensitive test can be obtained.

We next consider the three different types of nonlocality - Bell nonlocality, steering, and entanglement that may exist between two sites, as introduced by Wiseman and co-workers [21, 22, 38]. Following those authors, the fully separable LHV model becomes a quantum separable model when there exists a local quantum density operator $\rho_{j}^{\lambda}$ such that $\left\langle X_{j}\right\rangle_{\lambda}=\operatorname{Tr}\left(\rho_{j}^{\lambda} X_{j}\right)$, for each $j$. In this case, the system is described by a fully separable density matrix $\rho$ and failure of the model implies entanglement. To test for genuine $N$-partite entanglement, a partially separable model is used [11, 12], where $\rho=\sum_{s} P_{s} \int_{\lambda} d \lambda P_{s}(\lambda) \rho_{A_{s}}^{\lambda} \rho_{B_{s}}^{\lambda} d \lambda$ and $\rho_{A_{s}}^{\lambda}$ is a density operator, not necessarily factorisable, for the group $A_{s}$ (similarly $\rho_{B_{s}}^{\lambda}$ ). This is equivalent to the hybrid local-nonlocal model (1) but with the further constraint that moments for $A_{s}$ and $B_{s}$ each arise from a quantum density matrix, $\rho_{A_{s}}^{\lambda}$ and $\rho_{B_{s}}^{\lambda}$, respectively. The failure of all such models demonstrates genuine $N$-partite entanglement [13].

Now we turn to the case of steering. Following Ref. [21], we impose the asymmetric constraint on the model

\section{LHV}

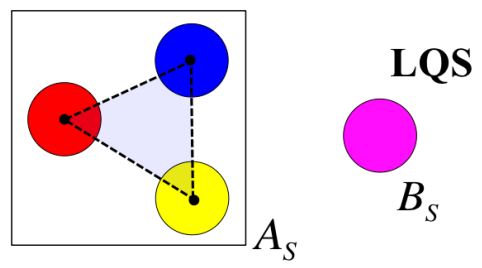

Figure 1. Depiction of one of the bipartitions $\left\{A_{s}, B_{s}\right\}_{s t}$ of the Hybrid Local Hidden State (LHS) local-nonlocal model for $N=4$. Here three sites can share a Bell nonlocality, but four cannot. The group $A_{s}$ "steers" system $B_{s}$, if this model fails, when it is also constrained that $B_{s}$ be consistent with a Local Quantum State.

(11) that there exists a quantum density operator $\rho_{B_{s}}^{\lambda}$ for the group of sites labelled $B_{s}$, but not for the group labelled $A_{s}$. Failure of this model, called a Local Hidden State (LHS) model, demonstrates "steering" of system $B_{s}$ by measurements performed on $A_{s}$ (Fig. 1) [21] (we will abbreviate, to say " $A_{s}$ steers $B_{s}$ "). Such steering can be confirmed through the violation of EPR-steering inequalities that are closely associated with the EPR paradox [28, 38]. A hierarchy of nonlocality is implied by the definitions. The local quantum state (LQS) description $\rho_{A_{s}}^{\lambda}$ is a particular example of a local hidden variable (LHV) one. Hence, Bell nonlocality between two groups $A_{s}$ and $B_{s}$ implies both steering and entanglement, and steering implies entanglement (but not Bell nonlocality). Unlike the other two nonlocalities, EPR steering is directional: that $A$ can steer $B$ does not imply that $B$ can steer $A$ [34].

A definition of genuine multi-partite steering follows naturally. Genuine tripartite steering exists iff it can be shown that a steering nonlocality is necessarily shared among all 3 sites. This means that the system cannot be described by any state in which steering is shared among two sites only. In this paper, we say two parties "share steering" if (at least) one can be shown to steer the other. The definition must be consistent with the operational definitions of Refs. [7, 8], namely, that steering shared among $N$ parties cannot be created by local operations and allowed classical communication (LOCC), even when $N-1$ parties collaborate. It will become necessary to constrain the collective hidden variable groups $A_{s}$ so that they satisfy no-signalling principles [7, 8].

However, we are able to use the partitioning of the model (1) to arrive at conditions that suffice to detect genuine multipartite steering. We now prove four results, which lead to simple criteria, and give properties that make multipartite EPR steering useful.

Result (1): Hybrid LHS local-nonlocal model: The failure of the model (10) where each group $B_{s}$ consists of one LQS site and group $A_{s}$ consists of 2 sites is sufficient to demonstrate genuine tripartite steering. 
Proof: It has been proved that the set of TOBL states are strictly contained within the set of BL states [7, 8]. Hence, falsification of all Svetlichny models (1) is sufficient to demonstrate genuine tripartite Bell nonlocality. The possible bipartitions $\left\{A_{s}, B_{s}\right\}$ are $\{\{1,2\}, 3\}$, $\{\{1,3\}, 2\}$ and $\{\{2,3\}, 1\}$. The model allows for Bell nonlocality between the sites of $A_{s}$. Hence, by the hierarchy of nonlocality, all ways in which steering can be shared between any two sites are described by the model. The failure of the model where site $B_{s}$ is a LQS is sufficient to imply steering, though not Bell nonlocality, between the groups $A_{s}$ and $B_{s}$. Thus, genuine tripartite steering is demonstrated if this model is falsified. The extension for larger $N$ is discussed in the Supplemental Material [44].

To falsify the model of Result (1), we need to rule out all mixtures of the relevant bipartitions (which we denote $\left\{A_{s}, B_{s}\right\}_{s t}$ ) that account for the way steering can be shared between 2 parties. The next result tells us how to rule out that the system can be described by any one of these bipartitions. This will prove genuine tripartite steering (and more generally $N$-partite steering), if we are constrained to pure state models, where mixtures of different bipartitions are not possible (a property that holds for pure quantum states). First, we introduce a definition: we demonstrate "collective $N$-partite steering of a system $B$ ", if it is shown that the steering of $B$ by a group of $N-1$ parties $A$ cannot also be demonstrated by the measurements of fewer than $N$ parties. As might be expected, we find a close relationship between collective $N$-partite steering, which always involves $N$ parties, and genuine $N$-partite steering.

Result (2): Pure-state genuine $N$-partite steering: (a) All bipartitions $\left\{A_{s}, B_{s}\right\}_{s t}$ of the model are negated, if it is shown that each group $\left\{A_{s}\right\}$ collectively steers $B_{s}$. (b) For the tripartite case, it is sufficient to show that each party can be steered by one or both of the other two.

Proof: (a) Take by example $N=4$. The possible bipartitions $\left\{A_{s}, B_{s}\right\}$ are $\{\{1,2,3\}, 4\},\{\{1,2\},\{3,4\}\}$, and all permutations. Steering of any one party $B_{s}$ by $A_{s}$ negates the first type of bipartition. The second type of partition is negated by the steering of $B_{s}$, that cannot be described as steering by only one other party. (b) Take each of $\{\{1,2\}, 3\},\{\{1,3\}, 2\}$ and $\{\{2,3\}, 1\}$. The first is negated if 3 is steered, the second if 2 is steered, the third if 1 is steered.

The Result (2) shows that collective steering is a type of genuine multipartite steering. If we demonstrate multipartite collective steering, then we confirm genuine multipartite steering, for the case of pure states.

The usefulness of this sort of genuine multipartite steering can be understood, once we realise the connection between steering and the EPR paradox 21, 29, 38]. It has been shown in Refs. [28, 31, 38] that steering of a single spin- $1 / 2$ qubit $B$ is confirmed, if a group of parties $A$ can infer, with sufficient accuracy, both of the Pauli spin components, $\sigma_{x}^{B}$ and $\sigma_{y}^{B}$. (They use different measurements for each inference). Similarly, steering of a single harmonic oscillator is confirmed, if the parties $A$ can infer accurately the values of both position and momentum, $x_{B}$ and $p_{B}$. Specifically, for any quantum state at $B$, the quantum uncertainty relations $\left(\Delta \sigma_{x}^{B}\right)^{2}+\left(\Delta \sigma_{y}^{B}\right)^{2} \geq 1$ and $\Delta x_{B} \Delta p_{B} \geq 1$ must hold [42] (we assume appropriate scaling). We confirm the steering of $B$ by $A$, if

$$
S_{B \mid A} \equiv \Delta_{i n f, A} x_{B} \Delta_{i n f, A} p_{B}<1
$$

where $\Delta_{i n f, A} x_{B}$ is the uncertainty in the prediction $x_{\text {pred }}$ of $x_{B}$ based on local measurements on system $A[21,38$, 43]. Alternatively, we confirm steering if

$$
\mathcal{S}_{B \mid A} \equiv\left(\Delta_{i n f, A} \sigma_{x}^{B}\right)^{2}+\left(\Delta_{i n f, A} \sigma_{y}^{B}\right)^{2}<1
$$

These inequalities have been used to confirm the EPR paradox, for bipartite systems [30].

Now we can establish a link with the quantum information protocol of "quantum secret sharing" [40, 41]. Suppose the parties at $A$ are shown to steer $B$, by measurements that reveal a reduced noise on the inferences, so that $S_{B \mid A}<1$. If it is known that group $A$ collectively steers $B$, then (by definition) the uncertainty product $S_{B \mid A}$ based on any measurements made by fewer parties at $A$ must exceed 1 . Thus, collective $N$-party steering provides the resource for " $N$-party quantum secret sharing", whereby $N-1$ parties must collaborate in order to deduce, by measurements on their systems, the value of the amplitude/ qubit of system $B$.

This motivates us to prove another useful result. A monogamy of EPR steering holds, that guarantees minimum noise levels on any inferences that could be made by "eavesdropping parties". The noise levels are a direct consequence of the quantum uncertainty relation of system $B$ only, and therefore provide "one-sided deviceindependent" security, whereby no assumptions are made about the exact nature of the strategies of the eavesdropping parties [25, 27]. Moreover, the amount of noise incurred is directly determinable from the degree of steering measured by the collaborating parties, $A$ and $B$.

Result (3): Steering and security: If it can be proved by violation of an EPR steering inequality involving two observables at each site that group $A$ steers $B$, then there can be no third group $C$ independent of $A$ that can also violate the same inequality. In particular, $S_{B \mid A} S_{B \mid C} \geq 1$.

Proof: Consider the EPR steering inequality $S_{B \mid A}<$ 1 defined above. Group $A$ performs measurements to predict $x_{B}\left(p_{B}\right)$ with uncertainty $\Delta_{i n f, A} x_{B}$ $\left(\Delta_{\text {inf }, A} p_{B}\right)$ while simultaneously $C$ can perform measurements to predict $p_{B}\left(x_{B}\right)$ with uncertainty $\Delta_{i n f, C} p_{B}$ $\left(\Delta_{i n f, C} x_{B}\right)$. The Heisenberg uncertainty principle implies $S_{B \mid A} S_{B \mid C} \geq 1$. More generally, the proofs follow because the state of $B$ conditioned on the joint outcomes of $\mathrm{A}$ and $\mathrm{C}$ is a quantum state. 
We return to the fundamental and challenging problem of detecting genuine $N$-partite steering. To do this, without the pure state assumption, we need to negate all mixtures of the possible bipartitions $\left\{A_{s}, B_{s}\right\}_{s t}$, that describe the possible ways to share steering among fewer than $N$ parties. For some systems, this can be done by violation of a single EPR steering inequality, and we give derivations in the Supplemental Materials [44]. Generally, however, it is enough to demonstrate "strong" EPR steering of each party, by the others.

Result (4): Genuine tripartite steering: Suppose $S_{i} \equiv S_{i \mid\{j k\}}<1$ indicates steering of party $i$ by the other parties $\{j, k\}$, where $S_{i \mid\{j k\}}$ is either the product or the sum of conditional inference variances, as defined for (2) and (3). Genuine tripartite steering is confirmed whenever $S_{1}+S_{2}+S_{3}<1$.

Proof: Suppose the system is a mixture of the three bipartitions $\{\{1,2\},\{3\}\}_{\text {st }}, \quad\{\{1,3\},\{2\}\}_{\text {st }}$ and $\{\{2,3\},\{1\}\}_{s t}$, which we label $s=3,2,1$ respectively, with relative probabilities $P_{s}$. For any such mixture: $S_{i} \geq \sum_{s} P_{s} S_{i, s}$ where $S_{i . s}$ denotes the value $S_{i}$ for a system in bipartition $s$ [42]. By definition of steering, $S_{i, i} \geq 1$. Hence, $S_{i} \geq P_{i}$, and the result follows on using $\sum_{s} P_{s}=1$.

$N$-partite GHZ qubit states: The $N$ - spin GHZ state $\frac{1}{\sqrt{2}}\left\{|\uparrow\rangle^{\otimes N}-|\downarrow\rangle^{\otimes N}\right\}$ predicts genuine $N$-partite steering. Here $|\downarrow\rangle_{j},|\uparrow\rangle_{j}$ are eigenstates of the Pauli spin $\sigma_{z}^{(j)}$ of the $j$ th particle. For example, for $N$ odd, the GHZ state is an eigenstate of $\sigma_{x}^{(N)} \prod_{j=1}^{N-1} \sigma_{y}^{(j)}$ (and all other products arising from the permutations among the $N$ sites) [9, 45]. Thus, any group $A$ of $N-1$ observers is able to predict the outcome of the spin $\sigma_{x}^{(N)}$ of the $N$ th particle, by measuring $\sigma_{x, p r e d}^{(N)}=(-1)^{(N+1) / 2} \prod_{j=1}^{N-1} \sigma_{y}^{(j)}$. In a similar way, the spin $\sigma_{y}$ of the $N$ th particle can be predicted if the $N-1$ observers measure the spin product $\sigma_{y, p r e d}^{(N)}=(-1)^{(N+1) / 2} \sigma_{x}^{(N-1)} \prod_{j=1}^{(N-2)} \sigma_{y}^{j}$. The variances $\left(\Delta_{i n f, A} \sigma_{x / y}^{(N)}\right)^{2}$ are zero for the GHZ state. In fact, from (3), we see that measurement of

$$
\mathcal{S}_{N}=\left(\Delta\left(\sigma_{x}^{(N)}-\sigma_{x, p r e d}^{(N)}\right)\right)^{2}+\left(\Delta\left(\sigma_{y}^{(N)}-\sigma_{y, p r e d}^{(N)}\right)\right)^{2}<1
$$

implies EPR steering of the $N$-th spin. For $N=3$, a measurement of $S_{j}<1 / 3$ for each $j=1,2,3$ would be sufficient to confirm genuine tripartite steering. Since two observables, $\sigma_{x}$ and $\sigma_{y}$, are involved, Result (3) applies, to ensure that the inferences of any third group of observers $C$ cannot satisfy (4).

EPR steering inequalities with three observables have recently enabled verification of steering in a "loopholefree" way without fair sampling assumptions [35 37. Motivated by this, we have derived a multipartite EPR steering inequality for 3 spins: $\left(\Delta_{i n f, A} \sigma_{x}^{B}\right)^{2}+$ $\left(\Delta_{i n f, A} \sigma_{y}^{B}\right)^{2}+\left(\Delta_{i n f, A} \sigma_{z}^{B}\right)^{2}<2$. We find the inequality is satisfied for GHZ states provided the overall detection efficiency of group $A$ exceeds $1 / 3$, making loophole-free
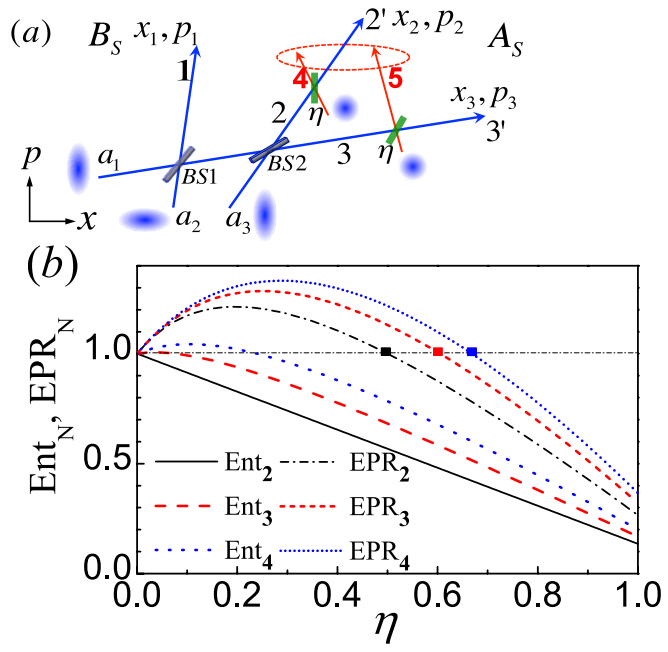

Figure 2. (a) Genuine CV tripartite entanglement for beams $\{1,2,3\}$ can be generated via squeezed states and beam splitters (BS) [17]. (b) Genuine tripartite entanglement exists for $\left\{1,2^{\prime}, 3^{\prime}\right\}$ when Ent $3<1$ whereas EPR steering of $\{1\}$ by $\left\{2^{\prime}, 3^{\prime}\right\}$ is confirmed when $E P R_{3}=\Delta_{\text {inf, } A^{\prime}} x_{1} \Delta_{\text {inf, } A^{\prime}} p_{1}<1$. Here, $\eta$ is the efficiency of transmission of the eavesdropping beam splitters. Cases $N=2,4$ are also shown. The loss of steering reveals the eavesdropper.

photonic demonstration experimentally feasible. Details are given in the Supplemental Materials [44].

$C V$ GHZ states: Consider $N$ harmonic oscillators (fields) at sites $j$, with boson operators $a_{j}$. The quadrature amplitudes $x_{j}, p_{j}$ are given by $a_{j}=x_{j}+i p_{j}$. A tripartite CV GHZ state is a simultaneous eigenstate of $x_{k}-x_{j}$ and $p_{1}+p_{2}+p_{3}$ with eigenvalues 0 [17]. For such a state: [46]:

$$
\begin{aligned}
& \left(\Delta_{i n f,\{k m\}} x_{j}\right)^{2}=\left(\Delta\left(x_{j}-x_{k}\right)\right)^{2}=\left(\Delta\left(x_{j}-x_{m}\right)\right)^{2}=0 \\
& \left(\Delta_{i n f,\{k m\}} p_{j}\right)^{2}=\left(\Delta\left(p_{j}+\left(p_{k}+p_{m}\right)\right)\right)^{2}=0
\end{aligned}
$$

where $j, k, m=1,2,3$ and $j \neq k \neq m$. Measurement of

$$
S_{j} \equiv \Delta\left(x_{j}-x_{k}\right) \Delta\left(p_{j}+\left(p_{k}+p_{m}\right)\right)<1
$$

will confirm EPR steering of system $j$, and, by Result (4), $S_{1}+S_{2}+S_{3}<1$ will confirm genuine tripartite steering.

Entanglement versus EPR steering: We point out that the properties discussed in this paper do not hold generally for multipartite entangled states. For example, consider the CV GHZ state depicted in Fig. 2a. This state shows collective steering, illustrated by the exact predictions for $x_{1}$ and $p_{1}$ by systems $A=\{2,3\}$, so that $\Delta_{i n f, A} x_{1} \Delta_{i n f, A} p_{1} \rightarrow 0$. If the beams 2 and 3 are coupled to 50:50 beam splitters, the collective steering necessarily vanishes. Two symmetric sets of beams exist in this case: $A^{\prime}=\left\{2^{\prime}, 3^{\prime}\right\}$ and an eavesdropping set, $E=\{4,5\}$. Hence, by Result $(3), \Delta_{i n f, A^{\prime}} x_{1} \Delta_{i n f, A^{\prime}} p_{1}=$ $\Delta_{i n f, E} x_{1} \Delta_{i n f, E} p_{1} \geq 1$. Yet, both sets remain genuine tripartite entangled with beam 1 (Fig. $2 \mathrm{~b}$ ), as measurable using the two-observable inequalities of Ref. [11, 47]. 
Hence, Result (3) does not hold for multipartite entanglement.

Conclusion: We have introduced the genuine tripartite EPR steering nonlocality, established its potential importance as a resource for secure quantum communications, and derived criteria that can be applied to current experiments. The observation of multipartite EPR steering in any of these systems would seem very feasible.

We thank A. Samblowski, S. Armstrong, Ping Koy Lam and P. Drummond for stimulating discussions. The work was suppported by Australian ARC Discovery Project and DECRA grants. Q. H. thanks support from China NNSF Grant No. 11121091.

[1] J. S. Bell, Physics 1, 195 (1964).

[2] G. Svetlichny, Phys. Rev. D 35, 3066 (1987).

[3] J. D. Bancal et al., Phys. Rev. Lett. 106, 020405 (2011). G. Adesso and S. Piano, arXiv:1307.3288v2.

[4] S. Ghose et al., Phys. Rev. Lett. 102, 250404 (2009).

[5] D. Collins et al., Phys. Rev. Lett. 88, 170405 (2002).

[6] M. Seevinck and G. Svetlichny, Phys. Rev. Lett. 89, 060401 (2002).

[7] R. Gallego et al., Phys. Rev. Lett., 109, 070401 (2012).

[8] J. Bancal et al., Phys Rev A 88, 014102 (2013).

[9] D. M. Greenberger, M. A. Horne and A. Zeilinger, in "Bell's Theorem, Quantum Theory, and Conceptions of the Universe" (Kluwer, Dordrecht, 1989), p. 69.

[10] J. Lavioe et al., New J. Phys 11, 073051 (2009). H. Lu et al., Phys. Rev. A 84, 012111 (2011).

[11] P. van Loock and A. Furusawa, Phys. Rev. A 67, 052315 (2003).

[12] R. Horodecki et al., Rev. Mod. Phys. 81, 865 (2009). O. Guhne and M. Seevinck, New J. Phys. 12, 053002 (2010).

[13] J. Bancal et al., Phys. Rev. Lett. 106, 250404 (2011).

[14] S. B. Papp et al., Science 324, 764 (2009).

[15] L. K. Shalm et al., Nat. Phys. 9, 19 (2013).

[16] D. Liebfried et al., Nature, 438, 639 (2005).

[17] T. Aoki et al., Phys. Rev. Lett. 91, 080404 (2003). B. Hage et al., Phys. Rev. A 81, 062301 (2010). P. van Loock and S. L. Braunstein, Phys. Rev. Lett. 84, 3482 (2000). P. van Loock and S.L. Braunstein, Phys. Rev. A 63, 022106 (2001).

[18] T. Monz et al., Phys. Rev. Lett. 106, 130506 (2011).

[19] S. Armstrong et al., Nature Commun. 3, 1026 (2012).

[20] R. F. Werner, Phys. Rev. A 40, 4277 (1989).

[21] H. M. Wiseman, S. J. Jones, and A. C. Doherty, Phys. Rev. Lett. 98, 140402 (2007).

[22] S. Jones et al., Phys. Rev. A 76, 052116 (2007).

[23] A. Cabello et al., Phys. Rev. Lett. 101, 120402 (2008).

[24] A. Ekert, Phys. Rev. Lett. 67, 661 (1991).

[25] A. Acin, N. Gisin and L. Masanes, Phys Rev. Lett. 97, 120405 (2006). A. Acin et al., Phys. Rev. Lett. 98, 230501 (2007).

[26] T. C. Ralph, Phys. Rev. A 61, 010303(R) (1999); V. Scarani et al., Rev. Mod. Phys. 81, 1301 (2009); Lars S. Madsen et al., Nature Commun. 3, 1083 (2012).

[27] C. Branciard et al., Phys. Rev. A 85, 010301(R) (2012).

[28] D. Saunders et al., Nat. Phys. 6, 845 (2010).

[29] A. Einstein, B. Podolsky, and N. Rosen, Phys. Rev. 47, 777 (1935).

[30] M. D. Reid et al., Rev. Mod. Phys. 81, 1727 (2009).

[31] E. G. Cavalcanti et al., Phys. Rev. A 84, 032115 (2011).

[32] Q. Y. He et al., Phys. Rev. A 83, 032120 (2011).

[33] T. Eberle et al., Phys. Rev. A 83, 052329 (2011). N. Takei et al., Phys. Rev. A 74, 060101(R) (2006); A. Samblowski et al., arXiv:1011.5766r2 [quant-ph]; S. Steinlechner, arXiv:1112.0461 v2 [quant-ph];

[34] V. Handchen et al., Nature Photonics 6, 596 (2012).

[35] D. H. Smith et al., Nat. Commun. 3, 625 (2012).

[36] B. Wittmann et al., New J. Phys. 14, 053030 (2012).

[37] A. J. Bennet et al., Phys. Rev. X 2, 031003 (2012).

[38] E. G. Cavalcanti et al., Phys. Rev. A. 80, 032112 (2009).

[39] H. J. Kimble, Nature 453, 1023 (2008).

[40] A Shamir, Commun. ACM 22, 612 (1979).

[41] M. Hillery et al., Phys. Rev. A 59, 1829 (1999). S. Gaertner et al., Phys. Rev. Lett. 98, 020503 (2007). A. M. Lance et al., New J. Phys. 5, 4 (2003). Y.-A. Chen et al., Phys. Rev. Lett. 95, 200502 (2005).

[42] H. F. Hofmann and S. Takeuchi, Phys. Rev. A 68, 032103 (2003). G. Toth, Phys. Rev. A 69, 052327 (2004).

[43] M. D. Reid, Phys. Rev. A 40, 913 (1989).

[44] Details are given in supplemental materials.

[45] N. D. Mermin, Phys. Rev. Lett. 65, 1838 (1990).

[46] M. K. Olsen et al., Journ Phys B: At. Mol. and Opt. 39, 2515 (2006).

[47] W. Bowen et al., Phys. Rev. Lett. 90, 043601 (2003). 